\title{
Primary Sjogren's syndrome and focal proliferative and crescentric necrotizing glomerulonephritis
}

\author{
Jordan Brodsky, Erin Patton, Ya Ju Chang \\ Department of Internal Medicine, Division of Rheumatology, Beth Israel Medical Center University Campus for the Albert Einstein \\ College of Medicine, New York, USA; Corresponding Author: Jordanbro@gmail.com
}

Received 17 March 2013; revised 20 April 2013; accepted 13 May 2013

Copyright (c) 2013 Jordan Brodsky et al. This is an open access article distributed under the Creative Commons Attribution License, which permits unrestricted use, distribution, and reproduction in any medium, provided the original work is properly cited.

\section{ABSTRACT}

Intro: Primary Sjogren's syndrome is a chronic systemic autoimmune disease. Although ocular and oral dryness are the hallmarks of this syndrome, renal involvement when present most commonly has the histological appearance of tubointerstitial nephritis and less often glomerular involvement. We report, a unique case of renal manifestations of primary Sjogren's syndrome with a proven histological focal proliferative and crescentric necrotizing glomeru-lonephritis. Case: A 30 years old man with Kimura disease presented with four months of subjective fevers, migrating skin erythema, dry mouth, and dry eyes. On further workup the patient was found to have a positive ANA, positive anti-SSA, and was evaluated by ophthalmology who found keratoconjunctivitis sicca. The patient was started on hydroxychloroquine in combination with prednisone. On follow up, the patient was found to be hypertensive with acute kidney injury, a creatinine of 3.65 , proteinuria and hematuria. Renal ultrasound was unremarkable. Cytoplasmic ANCA was positive and kidney biopsy was performed revealing non pauci-immune focal proliferative and necrotizing glomerulonephritis with cellular crescents and mesangial dense deposits by electron microscopy. Conclusion: To our knowledge, the association between biopsy proven focal proliferative and necrotizing glomerulonephritis with primary Sjogren's syndrome has never been previously described in the literature, nor has there been a reported case of association between Kimura disease, ANCA serology and primary Sjogren's syndrome.

Keywords: Sjogren's Syndrome; Focal Proliferative; Crescentric Necrotizing; Glomerulonephritis;

C-ANCA

\section{INTRODUCTION}

Primary Sjogren's syndrome is a chronic systemic autoimmune disease characterized by the presence of 2 of the following 3 criteria [1]: 1) positive serum anti-SSA and/or anti-SSB or positive rheumatoid factor (RF) and ANA titer $>1: 320,2$ ) ocular staining score $>3$ consistent with keratoconjunctivitis sicca, or 3) presence of focal lymphocytic sialadenitis with a focus score $>1$ focus/4 $\mathrm{mm}^{2}$ in labial salivary gland biopsy samples. Although ocular and oral dryness are the hallmarks of this syndrome, renal involvement when present most commonly has the histological appearance of tubointerstitial nephritis and less often glomerular involvement. We report a unique case of renal manifestations of primary Sjogren's syndrome with a proven histological focal proliferative and crescentric necrotizing glomerulonephritis.

\section{CASE}

A 30 years old man with a history of Kimura disease diagnosed by a lymph node biopsy showing marked eosinophilic infiltrate and a polymorphous infiltrate without clonality, presented to the primary care physician. The patient had two weeks of subjective fevers, migrating skin erythema, and pain in the knees, shoulder, lower back, and thigh. On review of systems, the patient reported having dry mouth and dry eyes for four months along with "bubbly urine". The patient denied any chest pain, shortness of breath, nausea, vomiting, headache, recent travel, illicit drug use, bug bites, or the use of prescription, over the counter, or herbal medications. Laboratory results revealed CRP 6, ESR 118, RF 106, ANA 1:640, anti SSA $>8$ and negative anti-SSB. Creatinine clearance was normal, however, urinalysis revealed 2+ protein with large blood. Vitals signs were significant for a blood pressure of 142/95. Physical exam was unremarkable except for bilateral shoulder pain on range of motion and dry eyes. Examination by ophthalmology 
confirmed the diagnosis of keratoconjunctivitis sicca with an ocular staining score $>3$. On further workup, C3 and C4 were decreased at $94 \mathrm{mg} / \mathrm{dL}$ and $8 \mathrm{mg} / \mathrm{dL}$ respectively. Anti ds-DNA antibody, ACE level, anti streplysin O antibody, anti Smith, anti RNP, anti CCP, Hepatitis B and $\mathrm{C}$ serology, serum cryoglobulins and RPR were all negative.

On follow-up, the rheumatologist noted palpable purpura on the extremities along with synovitis of the metacarpophalangeal joints bilaterally. Hydroxychloroquine $200 \mathrm{mg}$ daily in combination with prednisone $15 \mathrm{mg}$ twice daily was initiated. The patient was noncompliant with routine follow up and only after several months returned to the rheumatologist. At that time, the patient was found to be hypertensive with a blood pressure of $165 / 95$ and was in acute renal failure with proteinuria and hematuria on urinalysis. The patient was referred to a nephrologist for further workup.

Nephritic syndrome was diagnosed and blood work was repeated to determine the etiology revealing; Cytoplasmic ANCA (C-ANA) at 1:80, Proteinase-3 antibody at $>100 \mathrm{U} / \mathrm{mL}$, low C3 and C4, and a creatinine of 3.65 $\mathrm{mg} / \mathrm{dL}$. Ultrasound of the kidney was unremarkable and anti-glomerular basement membrane antibody was negative. Twenty-four hour urine revealed an elevated protein $2741 \mathrm{mg}$. Left kidney biopsy was performed and revealed focal proliferative and necrotizing glomerulonephritis with cellular crescents, glomeruli with partial fibrous crescents including subcapsular scar, global glomerulosclerosis, and interstitial nephritis. Interstitial fibrosis involved at least $50 \%$ of the cores (Figures 1 and 2). Electron microscopy revealed mesangial dense deposits. Direct immunofluorescence studies showed moderate amounts of IgG, IgM, C3, and questionable C1q and C4. There were no tubulo-reticular inclusions seen. The patient was started on oral cyclophosphamide and prednisone was increased to $30 \mathrm{mg}$ twice daily. Intravenous cyclophosphamide, Rituximab and solumedrol were refused by the patient.

\section{DISCUSSION}

Clinically significant renal involvement in patients with primary Sjogren's syndrome has been previously described only in isolated case reports and cohort studies. The pathogenesis of glomerulonephritis associated with primary Sjogren's syndrome is due to deposition of circulating immune complexes leading to membranous or membranoproliferative lesions. To our knowledge, this is the first reported case of focal proliferative and crescentric necrotizing glomerulonephritis with immune deposition.

Tubulointerstitial nephritis remains the most common presentation of renal involvement in primary Sjogren's syndrome [2-4] and is classically characterized by distal

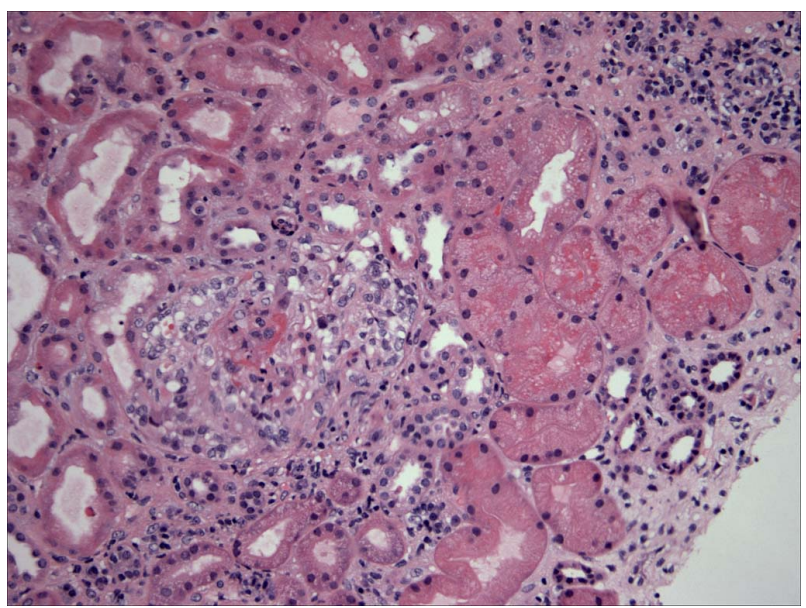

Figure 1. Low power magnification of kidney biopsy with focal proliferative and necrotizing glomerulonephritis with cellular crescents, glomeruli with partial fibrous crescents including subcapsular scar, global glomerulosclerosis, and interstitial nephritis.

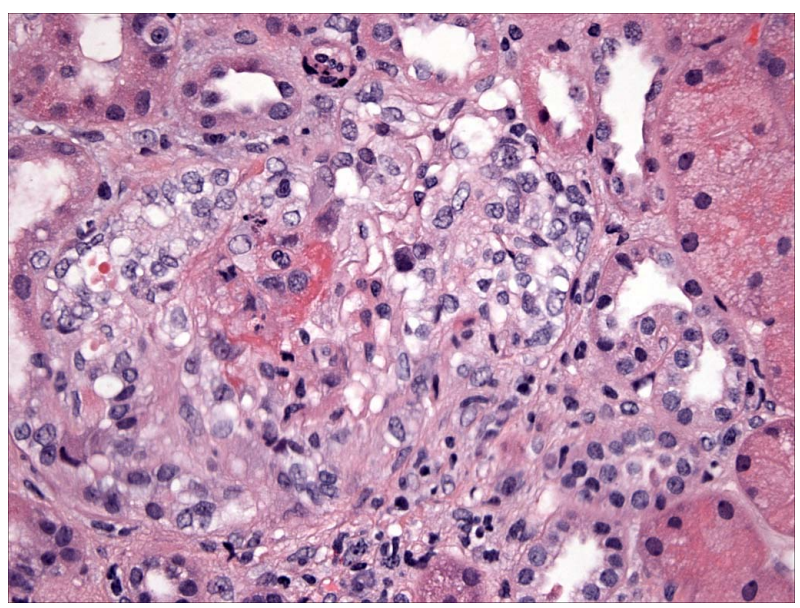

Figure 2. High power magnification of kidney biopsy of glomeruli with partial fibrous crescents including subcapsular scaring.

(type I) renal tubular acidosis and less commonly, proximal (type II) renal tubular acidosis (Fanconi syndrome). Glomerulonephritis associated with primary Sjogren's syndrome is a rare occurrence that tends to develop late in the course of the disease [5]. Case series [5-8] have described renal biopsies showing a wide variety of pathology including, membranoproliferative glomerulonephritis, mesangial proliferative glomerulonephritis, cryoglobulinemic glomerular nephritis, and tubulointerstitial nephritis.

The pathogenesis of glomerular disease in relationship to primary Sjogren's syndrome remains poorly understood. A study by Skopouli et al. [9] indicates that palpable purpura, decreased C4 complement concentration and mixed monoclonal cryoglobulinaemia are significant predictors for the development of glomerulonephritis in 
patients with primary Sjogren's syndrome. The pathogenesis of glomerulonephritis in the majority of these patients may be attributed to deposition of immune complexes formed by cryoprecipitating monoclonal IgM along with polyclonal IgG and IgA, however, glomerular disease may also occur in the absence of circulating cryoglobulins [10].

The clinical presenting symptoms of primary Sjogren's syndrome can mimic other connective tissue disease, particularly systemic lupus erythematosus. However, our patient did not fulfill the diagnostic criteria of systemic lupus erythematosus. In this case, the ANA was strongly positive, with a positive SSA and Rheumatoid Factor, but ds-DNA and Smith antibody were negative. The renal pathology in this case demonstrates focal proliferative and necrotizing glomerulonephritis with mesangial electron dense deposit and moderate amounts of IgG, IgM, C3, and questionable C4 and C1q. This is in contrast to lupus nephritis which classically stains highly positive for some mesangial changes with moderate amounts of IgG, IgA, IgM, C3, and C1q. The absence of anti ds-DNA antibody in addition to the lack of subendothelial deposition on electron microscopy makes it unlikely that glomerular disease was caused by systemic lupus erythematosus.

Typically P-ANCA positivity in patients with primary Sjogren's syndrome is associated with the presence of clinical manifestations attributable to vascular involveement, such as cutaneous vasculitis [11]. Episodes of primary Sjogren's syndrome associated with positive CANCA have only been described in a few cases, which have all been consistent with pauci-immune glomulonephritis characterized by normal compliment levels. In contrast, this case demonstrates C-ANCA positivity, low complement, and a renal biopsy revealing immune complex deposition. To our knowledge, this presentation has not been previously described in the literature. Additionally, extensive literature search revealed no association between Kimura disease and ANCA serology.

Kimura disease is a rare chronic inflammatory disorder of unknown etiology that causes painless unilateral inflammation of cervical lymph nodes and the major salivary glands, which become infiltrated by eosinophils and lymphocytes. Proteinuria has been reported in $12 \%$ to $16 \%$ of patients with Kimura disease, and $60 \%$ to $78 \%$ developed nephrotic syndrome [12-13]. Renal abnormalities such as membranous nephropathy, minimalchange disease, IgM, IgA, and IgE nephropathy, and mesangialproliferative glomerulonephritis may also develop in patients with Kimura disease. Kimura disease has not previously been described with focal proliferative and crescentric necrotizing glomerulonephritis. Therefore, this renal presentation is likely a new clinical manifestation of primary Sjogren's syndrome and not a direct effect of Kimura disease.

Further review of the literature failed to reveal any reported cases correlating Kimura disease and primary Sjogren's syndrome. The presentation of Kimura's disease is similar to Sjogrens syndrome in relationship to salivary gland involvement. However, the histology of the lymph node biopsy differentiates the two. In this case, lymph node biopsy was not consistent with primary Sjogren's syndrome as no lymphocytic aggregation was seen.

\section{CONCLUSION}

To our knowledge, the association between biopsy proven focal proliferative and necrotizing glomerulonephritis with primary Sjogren's syndrome has never been previously described in the literature, nor has there been a reported case of association between Kimura disease, ANCA serology and primary Sjogren’s syndrome.

\section{ACKNOWLEDGEMENTS}

Violette Ghali M.D. Department of Pathology. Beth Israel Medical Center University Campus for the Albert Einstein College of Medicine.

\section{REFERENCES}

[1] Shiboski, S.C., Shiboski, C.H., Criswell, A., et al. (2012) American college of rheumatology classification criteria for Sjögren's syndrome: A data-driven, expert consensus approach in the Sjögren's International Collaborative Clinical Alliance Cohort. Arthritis Care Research, 64, 475487. doi:10.1002/acr.21591

[2] Tsokos, M., Lazarou, S.A. and Moutsopoulos, H.M. (1987) Vasculitis in primary Sjogren's syndrome. Histologic classification and clinical presentation. American Journal of Clinical Pathology, 88, 26-31.

[3] Itzgerald, S., Hurst, N.P. and Pannall, P.R. (1987) Sjogren's syndrome, vasculitis, and cryoglobulinaemia associated with a monoclonal IgM (kappa) paraprotein with rheumatoid factor activity. Annals of the Rheumatic Disease, 46, 485-487. doi:10.1136/ard.46.6.485

[4] Moutsopoulos, H.M., Balow, J.E., Lawley, T.J., et al. (1978) Immune complex glomerulonephritis in sicca syndrome. American Journal of Medicine, 64, 955-960. doi:10.1016/0002-9343(78)90449-7

[5] Maripuri, S., Grande, J.P., Osborn, T.G., et al. (2009) Renal involvement in primary Sjögren's syndrome: A clinicopathologic study. Clinical Journal of the American Society of Nephrology, 4, 1423-1431. doi:10.2215/CJN.00980209

[6] Goules, A., Masouridi, S., Tzioufas, A.G., et al. (2000) Clinically significant and biopsy-documented renal involvement in primary Sjögren syndrome. Medicine (Baltimore), 79, 241-249. doi:10.1097/00005792-200007000-00005

[7] Bossini, N., Savoldi, S., Franceschini, F., et al. (2001) Clinical and morphological features of kidney involve- 
ment in primary Sjogren's syndrome. Nephrology Dialysis Transplantation, 16, 2328-2336.

doi:10.1093/ndt/16.12.2328

[8] Goules, A., Masouridi, S., Tzioufas, A.G., et al. (2000) Clinically significant and biopsy-documented renal involvement in primary Sjogren syndrome. Medicine (Baltimore), 79, 241-249.

doi:10.1097/00005792-200007000-00005

[9] Skopouli, F.N., Dafni, U. and Ioannidis, J.P.A. (2000) Clinical evolution and morbidity and mortality of primary Sjögren's syndrome. Seminars in Arthritis and Rheumatism, 29, 296-304. doi:10.1016/S0049-0172(00)80016-5

[10] Bossini, N., Savoldi, S., Franceschini, F., Mombelloni, S., et al. (2001) Clinical and morphological features of kidney involvement in primary Sjögren's syndrome. $\mathrm{Ne}$ phrology Dialysis Transplantation, 16, 2328-2336. doi:10.1093/ndt/16.12.2328

[11] Font, J., Ramos-Casals, M., Cervera, R., et al. (1998) Antineutrophil cytoplasmic antibodies in primary Sjögren's syndrome: Prevalence and clinical significance. Rheumatology, 37, 1287-1291. doi:10.1093/rheumatology/37.12.1287

[12] Matsuda, O., Makiguchi, K., Ishibashi, K., et al. (1992) Long term effects of steroid treatment on nephrotic syndrome associated with Kimura's disease and a review of the literature. Clinical Nephrology, 37, 119-123.

[13] Yamada, A., Mitsuhashi, K., Miyakawa, Y., et al. (1982) Membranous glomerulonephritis associated with eosinophilic lymphfolliculosis of the skin (Kimura's disease): Report of a case and review of the literature. Clinical Nephrology, 18, 211-215. 